University of Warwick institutional repository: http://go.warwick.ac.uk/wrap This paper is made available online in accordance with publisher policies. Please scroll down to view the document itself. Please refer to the repository record for this item and our policy information available from the repository home page for further information.

To see the final version of this paper please visit the publisher's website. Access to the published version may require a subscription.

Author(s): L. F. J. Piper, T. D. Veal, C. F. McConville, Hai Lu, and W. J. Schaff

Article Title: Origin of the n-type conductivity of $\operatorname{InN}$ : The role of positively charged dislocations

Year of publication: 2006

Link to published version: http://dx.doi.org/10.1063/1.2214156

Publisher statement: None 


\title{
Origin of the $n$-type conductivity of InN: The role of positively charged dislocations
}

\author{
L. F. J. Piper, T. D. Veal, and C. F. McConville \\ Department of Physics, University of Warwick, Coventry, West Midlands CV4 7AL, United Kingdom \\ Hai Lu and W. J. Schaff ${ }^{\text {a) }}$ \\ Department of Electrical and Computer Engineering, Cornell University, Ithaca, New York 14853
}

(Received 16 February 2006; accepted 5 May 2006; published online 21 June 2006)

\begin{abstract}
As-grown $\mathrm{InN}$ is known to exhibit high unintentional $n$-type conductivity. Hall measurements from a range of high-quality single-crystalline epitaxially grown $\mathrm{InN}$ films reveal a dramatic reduction in the electron density (from low $10^{19}$ to low $10^{17} \mathrm{~cm}^{-3}$ ) with increasing film thickness (from 50 to $12000 \mathrm{~nm}$ ). The combination of background donors from impurities and the extreme electron accumulation at InN surfaces is shown to be insufficient to reproduce the measured film thickness dependence of the free-electron density. When positively charged nitrogen vacancies $\left(V_{\mathrm{N}}^{+}\right)$along dislocations are also included, agreement is obtained between the calculated and experimental thickness dependence of the free-electron concentration. (C) 2006 American Institute of Physics. [DOI: $10.1063 / 1.2214156]$
\end{abstract}

As-grown InN always exhibits $n$-type conductivity, with unintentional free-electron concentrations as high as $10^{21} \mathrm{~cm}^{-3}$. . Many theoretical and experimental studies have focused on determining the major reason for the unintentional $n$-type conductivity of InN. ${ }^{1}$ However, no consensus has yet been reached. The traditional candidates fall into two categories: donor impurities and donor-type native defects. The impurities most commonly suggested as the primary cause of InN's $n$-type conductivity are oxygen ${ }^{2}$ and hydrogen. ${ }^{3}$ Among native defects, the nitrogen vacancy $\left(V_{\mathrm{N}}\right)$ has been found from theoretical calculations to be a donor ${ }^{2}$ and has also been suggested as the major reason for the high $n$-type conductivity. ${ }^{1}$ Self-interstitials and antisite defects are energetically unfavorable in InN due to the small lattice constant and the large-size mismatch between the cations and anions, respectively. ${ }^{2,4}$ More recently, surface electron accumulation has emerged as another factor contributing to the $n$-type conductivity in $\mathrm{InN}^{5,6}$

The purpose of this letter is to investigate the relative importance of these three contributions to the $n$-type conductivity of InN. To this end, calculations of the film thickness dependence of the free-electron concentration in $\mathrm{InN}$ are compared with Hall measurements of high-quality InN films grown by molecular-beam epitaxy (MBE). The observed dramatic reduction in carrier concentration with increasing film thickness is successfully modeled by considering a homogeneous background of donor impurities, a constant surface sheet density due to electron accumulation, and positively charged $V_{\mathrm{N}}$ along dislocations whose density declines exponentially away from the InN/buffer layer interface. While other possibilities cannot be entirely ruled out, this letter highlights the role dislocations and surface electron accumulation play in causing the variation of free-electron density with InN film thickness.

InN films were grown on sapphire substrates by MBE. ${ }^{7,8}$ The optimized growth method that results in the highest quality epitaxial InN films, with the lowest electron concen-

\footnotetext{
${ }^{a)}$ Electronic mail: wjs2@cornell.edu
}

trations, highest mobilities, and lowest dislocation densities, uses an AlN nucleation layer and a GaN buffer layer deposited prior to InN growth.

Figure 1 displays the variation in the free-electron concentration as a function of film thickness for a set of $\mathrm{InN}$ samples that were all grown under similar conditions. The electron density decreases by two orders of magnitude (low $10^{19}$ to low $10^{17} \mathrm{~cm}^{-3}$ ) as the film thickness is increased by almost four orders of magnitude $(50-12000 \mathrm{~nm})$. The respective Hall electron mobility (not shown) steadily increases by at least one order of magnitude $(\sim 100$ to $\left.\sim 2000 \mathrm{~cm}^{2} \mathrm{~V}^{-1} \mathrm{~s}^{-1}\right)$ for the same change in film thickness.

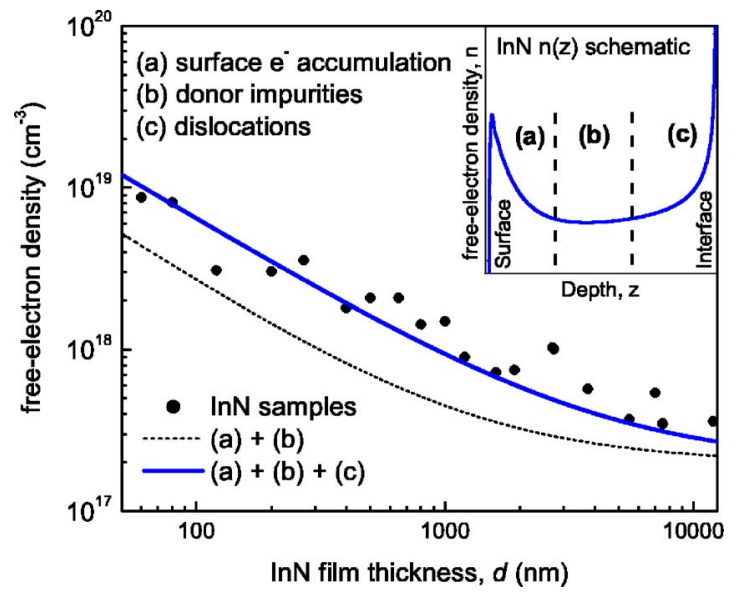

FIG. 1. (Color online) The free-electron density $n$ as a function of thickness for a range of InN films (dots). Two calculated free-electron density vs film thickness curves are also shown. The first (dashed black line) includes a uniform background free-electron density from impurity donors of 2 $\times 10^{17} \mathrm{~cm}^{-3}$ and a constant two-dimensional surface sheet density of 2.5 $\times 10^{13} \mathrm{~cm}^{-2}$. The second is the same but with the addition of free-electrons from positively charged dislocations (blue solid line). The inset is a schematic representation of the inhomogeneous electron distribution in an $\mathrm{InN}$ film. Three regions are identified: (a) where the surface electron accumulation layer is the major contribution to $n$, (b) a bulk layer where the electron density is mainly due to the background donor density from impurities, and (c) an interface region dominated by the contribution from the positively charged nitrogen vacancies along dislocations. 
Any explanation of the origin of the $n$-type conductivity in InN films must quantitatively account for this Hall data.

Donor impurities (e.g., $\mathrm{O}$ and $\mathrm{H}$ ) are the first possibility to consider to explain this film thickness dependence of the free-electron density in InN. This is because theoretical calculations have shown that the energy required for impurities to become donors is lower than the formation energy of native defect donors. ${ }^{2}$ High background impurity doping could be the major reason for the high $n$-type conductivity observed in some InN films when very high impurity densities are present. However, if InN films are grown without impurities being incorporated in significant concentrations, impurity-derived donors cannot be the main source of unintentional $n$-type doping. Indeed, secondary ion mass spectrometry (SIMS) of high-quality MBE-grown InN indicates that the concentration of impurities $(\mathrm{O}$ and $\mathrm{H})$ is too low to fully account for the measured free-electron densities. ${ }^{9}$ Furthermore, irrespective of the absolute concentrations of impurities (which can be difficult to reliably quantify by SIMS), SIMS results show that the impurity densities do not follow the trend of film thickness dependence exhibited by the free-electron concentration in Fig. 1. This suggests that impurities are not the major cause of $n$-type conductivity in these high-quality MBE-grown InN films.

The second phenomenon to consider to explain the film thickness dependence of InN's free-electron density is the native surface electron accumulation. Since this contributes a constant two-dimensional (2D) sheet free-electron density at the surface of each InN film irrespective of the film's thickness, it immediately offers one qualitative explanation of the film thickness dependence of the three-dimensional (3D) free-electron concentration. In order to obtain the experimental 3D free-electron densities $n_{\text {Hall }}^{3 \mathrm{D}}$, plotted in Fig. 1, the sheet density obtained from the Hall measurement is divided by the film thickness. For an electron accumulation layer, this means that, as the film thickness is increased, the fixed surface sheet density is numerically averaged over a greater depth, reducing the apparent 3D electron density. It must be emphasized that this does not in any way suggest that the charge associated with the electron accumulation is physically distributed through the film; it is localized within a few nanometers of the surface. ${ }^{6}$

While surface electron accumulation gives the correct trend with film thickness, its contribution to the free-electron density must be quantitatively evaluated against the experimental data in Fig. 1. Taking a constant surface sheet density of $n_{s}^{2 \mathrm{D}}=2.5 \times 10^{13} \mathrm{~cm}^{-2}$, in agreement with previous studies of InN surfaces, ${ }^{5,6}$ gives a value for $n_{\text {Hall }}^{3 \mathrm{D}}$ of $2.5 \times 10^{16} \mathrm{~cm}^{-3}$ for a $10000 \mathrm{~nm}$ thick film. This is more than an order of magnitude lower than the experimental value for this thickness $\left(3.5 \times 10^{17} \mathrm{~cm}^{-3}\right)$ shown in Fig. 1 .

This suggests that electron accumulation alone cannot fully account for the measured free-electron densities and that a low background concentration of donor impurities also contributes. Indeed, variable magnetic field Hall measurements of a thick InN film $(7.5 \mu \mathrm{m})$, analyzed by multiple carrier fitting and quantitative mobility spectrum analysis, indicated that the true bulk free-electron density (below the accumulation layer and far from the InN/buffer layer interface) is $\sim 2 \times 10^{17} \mathrm{~cm}^{-3} .{ }^{10}$ Therefore, any thickness dependence of the electron density must be superimposed on a uniform background of electrons from donor impurities. Therefore, for an InN film of thickness $d$, the Hall-measured Therefore, for an InN film of thickness $d$, the Hall-measured $\times 10^{10} \mathrm{~cm}^{-2}$. total sheet density $\left(n_{\mathrm{Hall}}^{3 \mathrm{D}} d\right)$ has been calculated as the sum of the sheet density arising from a uniform background of donor impurities $\left(n_{\text {imp }}^{3 \mathrm{D}} d\right)$ and the surface sheet density $\left(n_{s}^{2 \mathrm{D}}\right)$ resulting from the electron accumulation layer,

$$
n_{\text {Hall }}^{3 \mathrm{D}} d=n_{\mathrm{imp}}^{3 \mathrm{D}} d+n_{s}^{2 \mathrm{D}} .
$$

The Hall-derived 3D electron density is then given by dividing Eq. (1) by $d$,

$$
n_{\mathrm{Hall}}^{3 \mathrm{D}}=n_{\mathrm{imp}}^{3 \mathrm{D}}+\frac{n_{s}^{2 \mathrm{D}}}{d},
$$

where $n_{\text {imp }}^{3 \mathrm{D}}=2 \times 10^{17} \mathrm{~cm}^{-3}$ and $n_{s}^{2 \mathrm{D}}=2.5 \times 10^{13} \mathrm{~cm}^{-2}$. The calculated electron density variation with film thickness according to this model is shown in Fig. 1 (black dashed line). For this case, the calculated electron density is significantly lower than that observed for all film thicknesses.

This indicates that an additional film thicknessdependent phenomenon is required to completely reproduce the experimental data. The remaining possibility is a film thickness-dependent contribution from donor defects. The obvious candidate is defects associated with threading dislocations, whose origin is the large lattice mismatch between the epilayer and the buffer layer. The dislocation density in $\mathrm{InN}$ is assumed to vary in a similar manner to $\mathrm{GaN}$, since structurally these two materials are alike. In GaN, the dislocation density reduces exponentially with increasing distance from the buffer layer, as reported by Jasinski and Liliental-Weber. ${ }^{11}$ This variation is due to the annihilation and fusion of the dislocations with increasing growth. ${ }^{12}$ To account for the charge associated with the dislocations, a term has been added to Eq. (2), such that

$$
n_{\mathrm{Hall}}^{3 \mathrm{D}}=n_{\mathrm{imp}}^{3 \mathrm{D}}+\frac{n_{s}^{2 \mathrm{D}}}{d}+\frac{C}{d} \int_{0}^{d} D(x) d x
$$

where $C$ is a constant, $D(x)=A\left(10^{-\log _{10} x}\right.$ ) (for $x \geqslant 50 \mathrm{~nm}$ ) represents the exponential decay of the dislocation density $D$ (in $\mathrm{cm}^{-2}$ ) at distance $x$ from the interface, and $A$ is a constant. ${ }^{11}$ The few transmission electron microscopy (TEM) studies of InN films performed so far support this assumption. Dislocation densities in InN of $5.0 \times 10^{10} \mathrm{~cm}^{-2}$ (Ref. 3) and $2.2 \times 10^{10} \mathrm{~cm}^{-2}$ (Ref. 12) have been reported for 450 and $760 \mathrm{~nm}$ away from the interface, respectively. Additionally, a very recent cross sectional TEM study of the dislocation density in $\mathrm{InN}$ indicates an exponentional falloff with increasing distance from the InN/AIN interface. ${ }^{13}$ The total sheet density of electrons from donor $V_{\mathrm{N}}$ along dislocations in a film of thickness $d$ is determined by integrating the exponentially varying dislocation density over the entire film thickness and multiplying by the charge contribution per unit length of each dislocation $C$. The contributions from each depth are summed to determine the total contribution to the freeelectron density from dislocations. Using the same quantities for the background and electron accumulation terms as before, a charge contribution of one electron every $2 \mathrm{~nm}$ along each dislocation is sufficient to reproduce the experimental variation of electron density with InN film thickness, as shown in Fig. 1 (blue solid line). This one electron every $2 \mathrm{~nm}$ along each dislocation equates to a local free-electron density from dislocations of $1 \times 10^{17} \mathrm{~cm}^{-3}$ at the distance from the interface where the dislocation density is 2

o AlP license or copyright; see http://apl.aip.org/apl/copyright.jsp 


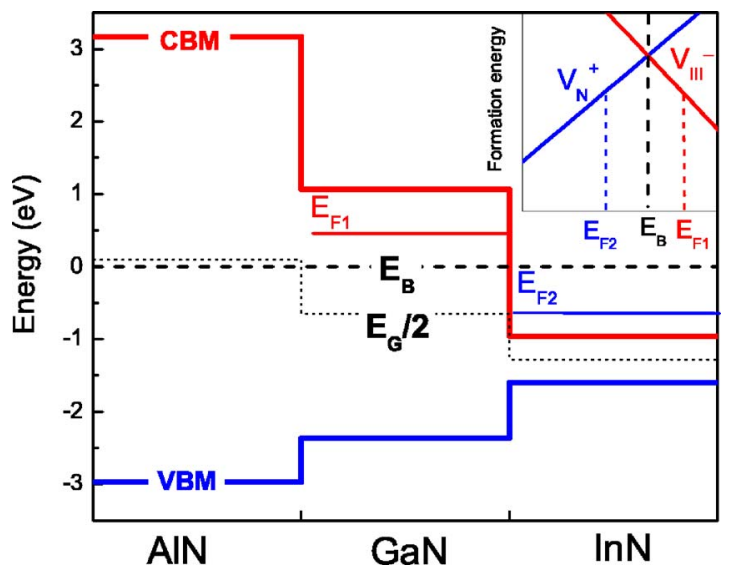

FIG. 2. (Color online) The calculated energetic positions of the $\Gamma$-point conduction band minimum (CBM) (red line) and valence band maximum (VBM) (blue line) of AlN, GaN, and $\mathrm{InN}$ with respect to the branch-point energy $\left(E_{B}\right)$ (dashed line) (Ref. 14) The midgap energy at the $\Gamma$ point is also shown (thin dotted line). The bulk Fermi levels for $n$-type $\mathrm{GaN}\left(E_{F 1}\right)$ and $n$-type $\operatorname{InN}\left(E_{F 2}\right)$ are also shown, above and below $E_{B}$, respectively. The inset schematically shows the variation of the formation energies with Fermi level of $V_{\mathrm{N}}^{+}$and $V_{\text {III }}$ for III-nitrides (after Ref. 18). The crossover between the two types of defects corresponds well to the location of $E_{B}$.

For this model to be plausible, the donor character of nitrogen vacancies in $n$-type $\mathrm{InN}$ must be explained. In $n$-type GaN, threading dislocations are negatively charged ${ }^{15}$ as a result of acceptor-type gallium vacancies $\left(V_{\mathrm{Ga}}\right)$ forming along the dislocation core. ${ }^{16}$ Studies of the effects of dislocations on the carrier mobility in GaN have indicated that approximately two acceptors per nanometer exist along the dislocation line. ${ }^{17}$ A previous InN study considered negatively charged acceptor dislocations by analogy with $\mathrm{GaN}^{3}{ }^{3}$ However, in view of the revision of the band gap since this work, positively charged donor nitrogen vacancies along dislocations are now considered to be energetically favorable for $n$-type InN.

Theoretical calculations of III-nitrides have revealed similarities between InN and GaN. ${ }^{18}$ Group III vacancies $\left(V_{\mathrm{III}}\right)$ become increasingly favorable as the Fermi level is increased with respect to the valence band maximum $(\mathrm{VBM})$. Conversely, $V_{\mathrm{N}}^{+}$becomes increasingly energetically favorable with decreasing Fermi level, as shown in the inset of Fig. 2. The crossover between the two types of defects corresponds well to the position of $E_{B}$ [for example, the VBM lies $2.4 \mathrm{eV}$ below $E_{B}$ for GaN (Ref. 14) in agreement with the crossover energy reported for GaN (Ref. 18)]. As described by the amphoteric defect model, ${ }^{19}$ the movement of the Fermi level away from $E_{B}$ lowers the formation energy of the type of defect required to move the Fermi level back towards $E_{B}$. Figure 2 shows the location of the $\Gamma$-point band extrema of III-nitrides with respect to the location of $E_{B}$. For $n$-type $\mathrm{GaN}$, the bulk Fermi level generally lies above $E_{B}$. Compensating $V_{\mathrm{Ga}}^{-}$becomes increasingly energetically favor- able as the $n$-type conductivity is increased. However, even for highly degenerate $n$-type InN, the bulk Fermi level lies far below $E_{B}$ and so $V_{\mathrm{N}}^{+}$are instead energetically favorable.

The variation of the Hall-measured electron concentration with increasing $\mathrm{InN}$ film thickness has been modeled by a constant background electron density (due to donor impurities), a fixed surface sheet density and the free electrons from $V_{\mathrm{N}}^{+}$along the dislocations. Therefore, impurities, native defects, and electron accumulation all play a significant role in producing the $n$-type conductivity of as-grown $\operatorname{InN}$. The existence of $V_{\mathrm{N}}^{+}$, donor impurities, and surface electron accumulation in $n$-type $\mathrm{InN}$ is due to the conduction band minimum (CBM) lying far below $E_{B}$. In this sense, the band structure of $\mathrm{InN}$ is the major reason for its $n$-type conductivity.

${ }^{1}$ A. G. Bhuiyan, A. Hashimoto, and A. Yamamoto, J. Appl. Phys. 94, 2779 (2003).

${ }^{2}$ C. Stampfl, C. G. Van de Walle, D. Vogel, P. Kruger, and J. Pollmann, Phys. Rev. B 61, R7846 (2000); the recent revision of the band gap of $\mathrm{InN}$ from 1.9 to $\sim 0.7 \mathrm{eV}$ changes the Fermi level at which $\mathrm{InN}$ changes from $p$-type to $n$-type. This makes $V_{\mathrm{N}}^{+}$more energetically favorable in $n$-type InN than originally reported by Stampfl et al. in 2000.

${ }^{3}$ D. C. Look, H. Lu, W. J. Schaff, J. Jasiniski, and Z. Liliental-Weber, Appl. Phys. Lett. 80, 258 (2002).

${ }^{4} \mathrm{C}$. G. Van de Walle, in Proceedings of the Tenth International Conference on Silicon Carbide and Related Materials, Lyon, France, 2003, edited by R. Madar, J. Camassel, and E. Blanquet (Trans Tech, Zurich, 2004); [Mater. Sci. Forum 457-460, 15 (2004)].

${ }^{5}$ H. Lu, W. J. Schaff, L. F. Eastman, and C. E. Stutz, Appl. Phys. Lett. 82, 1736 (2003).

${ }^{6}$ I. Mahboob, T. D. Veal, C. F. McConville, H. Lu, and W. J. Schaff, Phys. Rev. Lett. 92, 036804 (2004).

${ }^{7}$ W. J. Schaff, Hai Lu, L. F. Eastman, W. Walukiewicz, K. M. Yu, S. Keller, S. Kurtz, B. Keyes, and L. Gevilas, in State-of-the-Art Program on Compound Semiconductors XLI and Nitride and Wide Bandgap Semiconductors for Sensors, Photonics, and Electronics $V$, The Electrochemical Society Proceedings Series Vol. 2004-06, edited by H. M. Ng and A. G. Baca (Electrochemical Society, New York, 2004), pp. 358-371.

${ }^{8}$ K. M. Yu, Z. Liliental-Weber, W. Walukiewicz, W. Shan, J. W. Ager III, S. X. Li, R. E. Jones, E. E. Haller, H. Lu, and W. J. Schaff, Appl. Phys. Lett. 86, 071910 (2005).

${ }^{9}$ J. Wu, W. Walukiewicz, S. X. Li, R. Armitage, J. C. Ho, E. R. Weber, E. E. Haller, Hai Lu, W. J. Schaff, A. Barcz, and R. Jakiela, Appl. Phys. Lett. 84, 2805 (2003).

${ }^{10}$ C. H. Swartz, R. P. Tomkins, N. C. Giles, T. H. Myers, H. Lu, W. J. Schaff, and L. F. Eastman, J. Cryst. Growth 269, 29 (2004).

${ }^{11}$ J. Jasinski and Z. Liliental-Weber, J. Electron. Mater. 31, 429 (2002).

${ }^{12}$ C. J. Lu, L. A. Bendersky, H. Lu, and W. J. Schaff, Appl. Phys. Lett. 83, 2817 (2003).

${ }^{13}$ V. Lebedev, V. Cimalla, J. Pezoldt, M. Himmerlich, S. Krishok, J. A. Schaefer, O. Ambacher, F. M. Morales, J. G. Lozano, and D. Gonzalez (unpublished).

${ }^{14}$ W. Monch, J. Appl. Phys. 80, 5076 (1996).

${ }^{15}$ D. C. Look and J. R. Szelove, Phys. Rev. Lett. 82, 1237 (1999).

${ }^{16}$ A. F. Wright and U. Grossner, Appl. Phys. Lett. 73, 2751 (1998).

${ }^{17}$ H. M. Ng, D. Doppalapudi, T. D. Moustakas, N. G. Weimann, and L. F. Eastmann, Appl. Phys. Lett. 73, 821 (1998).

${ }^{18}$ C. G. Van de Walle and J. Neugebauer, J. Appl. Phys. 95, 3851 (2004).

${ }^{19}$ W. Walukiewicz, Appl. Phys. Lett. 54, 2094 (1989). 